\title{
Correlated Electron Microscopy across Length Scales to Elucidate Structural, Electrical and Chemical Properties of Oxide Grain Boundaries
}

\author{
William J. Bowman ${ }^{1,2}$, Madeleine N. Kelly ${ }^{3}$, Gregory S. Rohrer ${ }^{3}$, Cruz A. Hernandez ${ }^{1}$, Amith Darbal ${ }^{4}$ \\ and Peter A. Crozier ${ }^{1}$ \\ 1. School for Engineering of Matter, Transport and Energy, Arizona State University, Tempe, AZ, USA \\ 2. Laboratory for Electrochemical Interfaces, MIT, Cambridge, MA, US \\ 3. Department of Materials Science and Engineering, Carnegie Mellon University, Pittsburgh, PA, USA \\ 4. AppFive LLC, Tempe, AZ, USA
}

Because of their favorable ionic and/or electronic conductivity, non-stoichiometric oxides are utilized for energy storage, energy conversion, sensing, catalysis, gas separation, and information technologies, both potential and commercialized. Charge transport in these materials is influenced strongly by grain boundaries, which exhibit fluctuations in composition, chemistry and atomic structure within Ångstroms or nanometers [1-3]. Here, studies are presented that elucidate the interplay between macroscopic electrical conductivity, microscopic character, and local composition and electronic structure of grain boundaries in polycrystalline $\mathrm{CeO}_{2}$-based solid solutions. Electron energy-loss spectroscopy (EELS) in the aberration-correction scanning transmission electron microscope (AC-STEM) is used to quantify local composition and electronic structure. Electron diffraction orientation imaging microscopy is employed to assess microscopic grain boundary character, and links macro- and nanoscopic techniques. These correlated experimental approaches provide unique insights into fundamental GB science, and highlights how novel aspects of nanoscale GB engineering may be manipulated to control ion transport properties in electroceramics.

In the model system $\mathrm{Ca}_{\mathrm{x}} \mathrm{Ce}_{1-\mathrm{x}} \mathrm{O}_{2-\mathrm{x}}$, the enhancement of oxygen ionic conductivity by over two orders of magnitude is explicitly shown to result from modulation of the local composition of a GB layer or complexion a few nanometers in width [3]. EBSD from a series of polycrystalline samples with varying nominal $\mathrm{Ca}$ concentration (see Fig 1c,d for example) showed that all samples possessed random grain boundary character. AC-STEM EELS analysis demonstrated that local GB composition (see Fig. 1a,b), rather than structural GB character, primarily regulates ionic conductivity. In another solid solution $(\mathrm{Pr}, \mathrm{Gd})_{\mathrm{x}} \mathrm{CeO}_{2-\delta}(\mathrm{PGCO}), \mathrm{GB}$ conductivity was nearly 100 times greater than expected and a factor of four enrichment of Pr concentration was observed at the grain boundary, which suggested electronic conduction via small polaron hopping that was cited as the origin of the enhanced conductivity. This inspired the development of an approach to elucidate the effect of Pr enrichment on GB conductivity. STEM nanodiffraction orientation imaging (Fig. 2a) coupled with AC-STEM EELS was employed to estimate the composition of the entire GB population in a polycrystalline material. These compositional data were the input to a thermodynamic model used to predict electrical properties of the GB population, Fig. 2b [4]. These results suggest improved DC ionic conduction and enhanced electronic (small polaron) conduction under AC conditions.

\section{References:}

[1] X. Guo, R. Waser. Prog. Mater. Sci., 51 (2006) 151-206.

[2] A. Jasper, J.A. Kilner, D.W. McComb. Solid State Ion. 179 (2008) 904-908.

[3] W.J. Bowman, M.N. Kelly, G.S. Rohrer, C.A. Hernandez, P.A. Crozier. (In review). 
[4] W.J. Bowman, A. Darbal, P.A. Crozier, (In preparation).

[5] W.J.B. acknowledges the NSF's Graduate Research Fellowship (DGE-1211230) for financial support. M.N.K. and G.S.R. acknowledge support from the ONR-MURI program (grant no. N00014-110678). C.A.H. acknowledges ASU's Fulton Undergraduate Research Initiative for financial support.

P.A.C. and W.J.B. acknowledge support of NSF grant DMR-1308085. The authors acknowledge access to ASU's John M. Cowley Center for High Resolution Electron Microscopy.
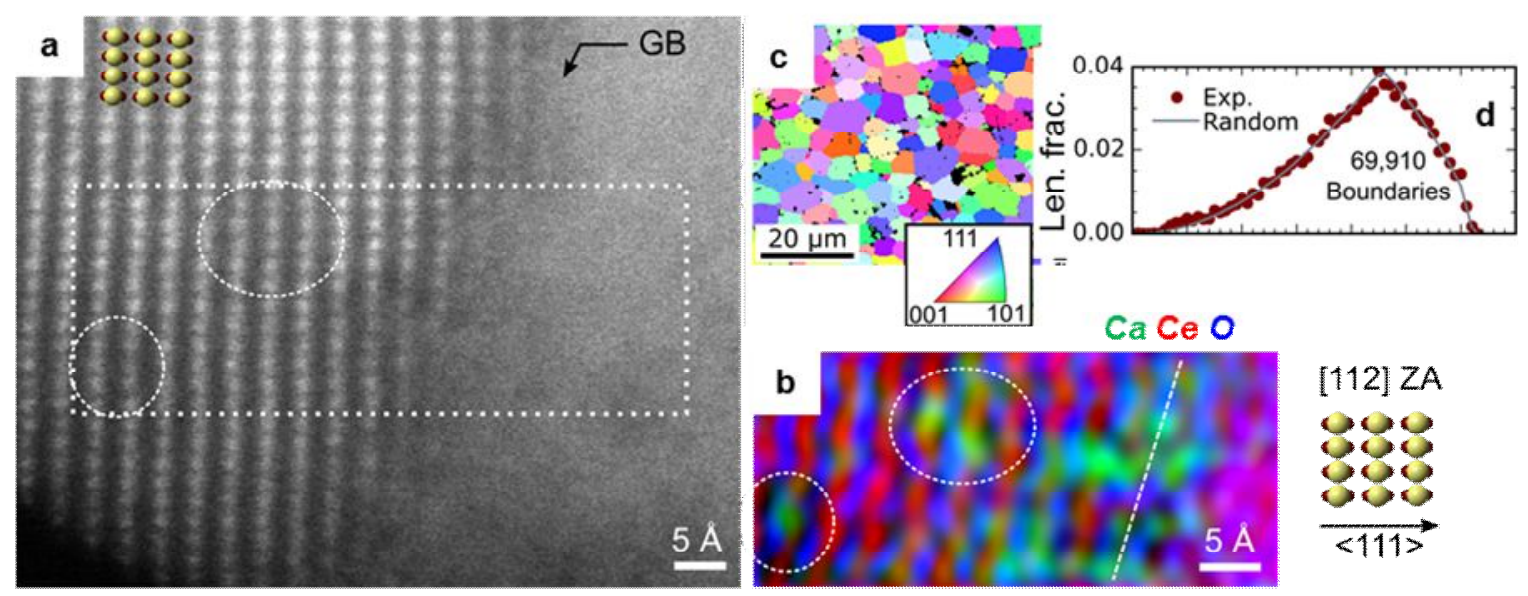

Figure 1. (c) AC-STEM ADF image of a $\mathrm{GB}$ in $\mathrm{Ca}_{0.1} \mathrm{Ce}_{0.9} \mathrm{O}_{1.9-\delta}$ with crystal model overlaid (at upper left) illustrating $\mathrm{CeO}_{2}$ observed along the [112] zone axis ( $\mathrm{Ce}=$ Yellow; $\left.\mathrm{O}=\mathrm{Red}\right)$. (b) Associated EELS elemental maps acquired in the region of (a) indicated with a dashed box; overlaid jump ratio maps following Fourier filtering using (111) diffraction spots, see crystal model annotations at right. Bending distortion in atomic planes is due to specimen drift/charging; data from JEOL ARM 200F. (c) SEM EBSD inverse pole figure map from $\mathrm{Ca}_{0.02} \mathrm{Ce}_{0.98} \mathrm{O}_{1.98-\delta}$ and (d) misorientation angle distribution (grain boundary length fraction vs. misorientation angle) showing random grain boundary orientation in these sintered oxides.
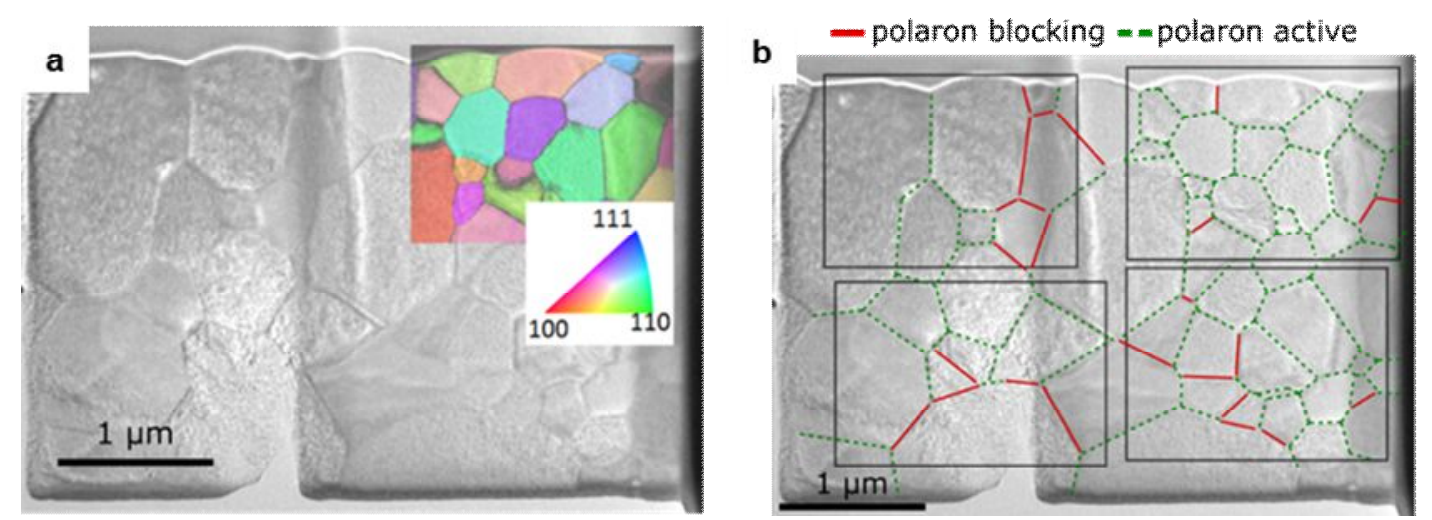

Figure 2. (a) Bright field TEM image of PGCO with overlaid inverse pole figure; grain orientation is indicated via color code corresponding to inset stereographic triangle. (b) Bright field TEM image of PGCO with regions analyzed using PEND outlined. GB segments are highlighted to indicate predicted small polaron transport behavior based on their $\operatorname{Pr}$ concentration: 'Polaron active' GBs have $[\mathrm{Pr}] \geq 10 \mathrm{~mole} \%$, and 'polaron blocking boundaries have $[\mathrm{Pr}]<10$ mole\%. Data from JEOL ARM $200 \mathrm{~F}$. 